\title{
PROJECTION OPERATOR TECHNIQUES IN LAGRANGIAN MECHANICS: SYMMETRICALLY COUPLED OSCILLATORS
}

\author{
J.N. BOYD and P.N. RAYCHOWDHURY \\ Department of Mathematical Sciences \\ Virginia Commonwealth University \\ Richmond, Virginia 23284 \\ U.S.A.
}

(Received July 6, 1979)

ABSTRACT. We apply projection operator techniques to the computation of the natural frequencies of oscillation for three symmetrically coupled mechanical systems. In each case, the rotation subgroup of the full symmetry group is used to determine the projection operators with the result that the Lagrangian must be expressed in terms of complex-valued coordinates. In the coordinate system obtained from the action of the projection operators upon the original coordinates, the Lagrangian yields equations of motion which are separated to the maximum extent made possible by symmetry considerations.

KEY WORDS AND PHRASES. Group representations, Harmonic oscillators, Lagrangian mechanics, Natural frequencies, Projection operators. 1980 MATHEMATICS SUBJECT CLASSIFICATION CODES. $20035,70 J 10$.

1. INTRODUCTION.

We consider a vibrating mechanical system of point masses and ideal rotators. 
If we define the n-dimensional column vector, $X$, by the notation $x \equiv \operatorname{col}\left(x_{1} x_{2} \cdots x_{n}\right)$, we are able to write the Lagrangian for the system as

$$
\mathrm{L}=\frac{1}{2} \mathrm{~m} \tilde{\mathrm{X}} \mathrm{T} \dot{\mathrm{X}}-\frac{1}{2} \mathrm{k} \tilde{\mathrm{X}} \mathrm{XX}
$$

where $\tilde{x}$, the transpose of $x$, is the row vector $\tilde{x} \equiv\left(x_{1} x_{2} \cdots x_{n}\right)$. Here we have $\dot{x}_{1}, \dot{x}_{2}, \cdots, \dot{x}_{n}$ and $x_{1}, x_{2}, \cdots, x_{n}$ as velocities and displacements with respect to an orthonormal set of vectors, $\left\{\hat{u}_{1}, \hat{u}_{2}, \cdots, \hat{u}_{n}\right\}$. Both $T$ and $v$ are symmetric $\mathrm{n} \times \mathrm{n}$ matrices with all entries real.

Let $\mathrm{G}$ be the finite point symmetry group of the mechanical system, and let us denote the distinct, nonequivalent, unitary matrix representations of G by $\Gamma^{(\mu)}(G)$, where $\mu \varepsilon\{1,2, \cdots, k\}$ and $k$ is the number of conjugacy classes in $G$. Then for $g \in G$, let us denote the $i, j$-th complex entry in the matrix $\Gamma^{(\mu)}(g)$ by $\Gamma_{i j}{ }^{(\mu)}(g)$.

Further, let $\phi(g)$ be the linear operator induced by $g \in G$ acting on the solution space which has basis $\left\{\hat{u}_{1}, \hat{u}_{2}, \cdots, \hat{u}_{n}\right\}$. Then we are in a position to define the projection operator

$$
P_{i j}{ }^{(\mu)}=\sum_{g \varepsilon G} \Gamma_{i j}{ }^{(\mu)}(g) \phi(g) .
$$

There are exactly $|\mathrm{G}|$ of these operators [4], [5].

The action of these operators on the basis $\left\{\hat{u}_{1}, \hat{u}_{2}, \cdots, \hat{u}_{n}\right\}$ will produce a new basis of symmetry coordinates with which it is possible to exploit the geometric symmetries of the system to the maximum extent in the solution of the equations of motion [6] . That is, from the new coordinates, we construct a unitary matrix $U$ such that $U_{T U}^{-1}$ and $U V U^{-1}$ will assume block diagonal forms which lead to significant simplifications in the equations of motion. In general, the entries in $U$ will be complex, leading to transformed velocities and displacements with real and imaginary components. 
That is,

$$
\begin{aligned}
\mathrm{L} & =\frac{1}{2} \mathrm{~m} \tilde{\dot{\mathrm{X}}} \mathrm{U}^{-1} \mathrm{UTU}^{-1} \mathrm{UX}-\frac{1}{2} \mathrm{k} \tilde{\mathrm{XU}}^{-1} \mathrm{UVU}^{-1} \mathrm{UX} \\
& =\frac{1}{2} \mathrm{~m} \tilde{\dot{N}} \mathrm{UTU}^{-1} \dot{\mathrm{N}}^{\star}-\frac{1}{2} \mathrm{k} \tilde{\mathrm{N} U V U}{ }^{-1} \mathrm{~N}^{\star}
\end{aligned}
$$

where $N \equiv \operatorname{col}\left(n_{1} n_{2} \cdots \eta_{n}\right)$. Note that $\dot{\eta}_{j}$ and $\eta_{j} \varepsilon C$ and that they give the velocity and displacement with respect to the symmetry coordinate $\hat{e}_{j}[1],[2]$. Also observe that $\eta_{j}{ }^{*}$ denotes the complex conjugate of $\eta_{j}$.

The equations of motion are then given by

$$
\frac{d}{d t}\left(\frac{\partial L}{\partial \eta_{j}}\right)-\frac{\partial L}{\partial \eta_{j}}=0 \text { or, equivalently, }
$$

by

$$
\frac{d}{d t}\left(\frac{\partial L}{\partial \eta_{j}^{*}}\right)-\frac{\partial L}{\partial \eta_{j}^{\star}}=0
$$

The use of the Lagrangian in the case that the new coordinates are complex is both convenient and intrinsically interesting.

\section{EXAMPLES.}

We now give three problems to illustrate the technique.

EXAMPLE 1. Six point masses of which three have the value $m$ and three have the value $M$ are symmetrically arranged about a fixed circle as shown below. They are interconnected with identical springs of force constant $k$. We desire to find the natural frequencies of vibrations for this system if all motion is of very small amplitude and is confined to the fixed circle.

SOLUTION. We take the symmetry group to be $C_{3}=\left\{\mathrm{E}, \mathrm{R}\left(120^{\circ}\right), \mathrm{R}\left(240^{\circ}\right)\right\}$ which is a subgroup of the full symmetry group $C_{3 v}$.

The irreducible representations over $\mathbb{C}$ are given by 


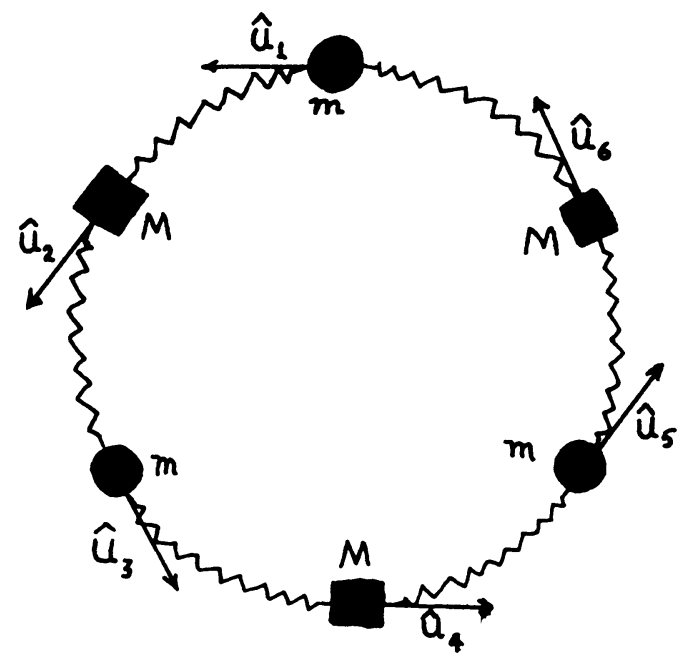

$$
\begin{aligned}
\Gamma^{(1)}\left(C_{3}\right): & \Gamma^{(1)}(g)=1 \forall g \varepsilon C_{3} . \\
\Gamma^{(2)}\left(C_{3}\right): & \Gamma^{(2)}(E)=1, \Gamma^{(2)}\left(R\left(120^{\circ}\right)\right)=-\frac{1}{2}+\frac{i \sqrt{3}}{2}, \\
& \Gamma^{(2)}\left(R\left(240^{\circ}\right)\right)=-\frac{1}{2}-\frac{i \sqrt{3}}{2} . \\
\Gamma^{(3)}\left(C_{3}\right): & \Gamma^{(3)}(E)=1, \Gamma^{(3)}\left(R\left(120^{\circ}\right)\right)=-\frac{1}{2}-\frac{i \sqrt{3}}{2}, \\
& \Gamma^{(3)}\left(R\left(240^{\circ}\right)\right)=-\frac{1}{2}+\frac{i \sqrt{3}}{2} .
\end{aligned}
$$

There are three projection operators which act upon the basis of unit tangents.

$$
\begin{aligned}
& \mathrm{P}_{11}^{(1)}=\sum_{g \in C_{3}} \Gamma{ }^{(1)}(\mathrm{g}) \phi(\mathrm{g}), \\
& \mathrm{P}_{11}^{(2)}=\sum_{\mathrm{g} \varepsilon \mathrm{C}_{3}} \Gamma{ }^{(2)}(\mathrm{g}) \phi(\mathrm{g}), \text { and } \\
& \mathrm{P}_{11}^{(3)}=\sum_{\mathrm{g} \varepsilon \mathrm{C}_{3}} \Gamma{ }^{(3)}(\mathrm{g}) \phi(\mathrm{g}) \text { where } \phi(\mathrm{g})
\end{aligned}
$$


is the linear operator corresponding to the symmetry element $g$. By computing the effects of the projection operators upon the unit tangents, $\hat{u}_{j}$, we form a new basis for our solution space and we can construct the unitary matrix $U$ with which we shall transform the Lagrangian.

$$
\begin{aligned}
& \mathrm{P}_{11}^{(1)} \hat{\mathrm{u}}_{1}=\hat{\mathrm{u}}_{1}+\hat{\mathrm{u}}_{3}+\hat{\mathrm{u}}_{5} \\
& \mathrm{P}_{11}^{(1)} \hat{\mathrm{u}}_{2}=\hat{\mathrm{u}}_{2}+\hat{\mathrm{u}}_{4}+\hat{\mathrm{u}}_{6} \\
& \mathrm{P}_{11}^{(2) \hat{\mathrm{u}}_{1}}=\hat{\mathrm{u}}_{1}+\left(-\frac{1}{2}-\frac{i \sqrt{3}}{2}\right) \hat{\mathrm{u}}_{3}+\left(-\frac{1}{2}+\frac{i \sqrt{3}}{2}\right) \hat{\mathrm{u}}_{5} \\
& \mathrm{P}_{11}^{(2)} \hat{\mathrm{u}}_{2}=\hat{\mathrm{u}}_{2}+\left(-\frac{1}{2}-\frac{i \sqrt{3}}{2}\right) \hat{\mathrm{u}}_{4}+\left(-\frac{1}{2}+\frac{i \sqrt{3}}{2}\right) \hat{\mathrm{u}}_{6} \\
& \mathrm{P}_{11}^{(3)} \hat{\mathrm{u}}_{1}=\hat{\mathrm{u}}_{1}+\left(-\frac{1}{2}+\frac{i \sqrt{3}}{2}\right) \hat{\mathrm{u}}_{3}+\left(-\frac{1}{2}-\frac{i \sqrt{3}}{2}\right) \hat{\mathrm{u}}_{5} \\
& \mathrm{P}_{11}^{(3)} \hat{\mathrm{u}}_{2}=\hat{\mathrm{u}}_{2}+\left(-\frac{1}{2}+\frac{i \sqrt{3}}{2}\right) \hat{\mathrm{u}}_{4}+\left(-\frac{1}{2}-\frac{i \sqrt{3}}{2}\right) \hat{u}_{6}
\end{aligned}
$$

We construct the matrix $U$ after normalizing the six orthogonal vectors above.

$$
\mathrm{U}=\frac{1}{\sqrt{3}}\left(\begin{array}{cccccc}
1 & 0 & 1 & 0 & 1 & 0 \\
0 & 1 & 0 & 1 & 0 & 1 \\
1 & 0 & \left(-\frac{1}{2}-\frac{i \sqrt{3}}{2}\right) & 0 & \left(-\frac{1}{2}+\frac{i \sqrt{3}}{2}\right) & 0 \\
0 & 1 & 0 & \left(-\frac{1}{2}-\frac{i \sqrt{3}}{2}\right) & 0 & \left(-\frac{1}{2}+\frac{i \sqrt{3}}{2}\right) \\
1 & 0 & \left(-\frac{1}{2}+\frac{i \sqrt{3}}{2}\right) & 0 & \left(-\frac{1}{2}-\frac{i \sqrt{3}}{2}\right) & 0 \\
0 & 1 & 0 & \left(-\frac{1}{2}+\frac{i \sqrt{3}}{2}\right) & 0 & \left(-\frac{1}{2}-\frac{i \sqrt{3}}{2}\right)
\end{array} \mid\right.
$$

and 


$$
U^{-1}=\frac{1}{\sqrt{3}}\left(\begin{array}{cccccc}
1 & 0 & 1 & 0 & 1 & 0 \\
0 & 1 & 0 & 1 & 0 & 1 \\
1 & 0 & \left(-\frac{1}{2}+\frac{i \sqrt{3}}{2}\right) & 0 & \left(-\frac{1}{2}-\frac{i \sqrt{3}}{2}\right) & 0 \\
0 & 1 & 0 & \left(-\frac{1}{2}+\frac{i \sqrt{3}}{2}\right) & 0 & \left(-\frac{1}{2}-\frac{i \sqrt{3}}{2}\right) \\
1 & 0 & \left(-\frac{1}{2}-\frac{i \sqrt{3}}{2}\right) & 0 & \left(-\frac{1}{2}+\frac{i \sqrt{3}}{2}\right) & 0 \\
0 & 1 & 0 & \left(-\frac{1}{2}-\frac{i \sqrt{3}}{2}\right) & 0 & \left(-\frac{1}{2}+\frac{i \sqrt{3}}{2}\right)
\end{array} \mid\right.
$$

The kinetic energy matrix is

$\mathrm{T}=\left(\begin{array}{cccccc}1 & 0 & 0 & 0 & 0 & 0 \\ 0 & \mathrm{M} / \mathrm{m} & 0 & 0 & 0 & 0 \\ 0 & 0 & 1 & 0 & 0 & 0 \\ 0 & 0 & 0 & \mathrm{M} / \mathrm{m} & 0 & 0 \\ 0 & 0 & 0 & 0 & 1 & 0 \\ 0 & 0 & 0 & 0 & 0 & \mathrm{M} / \mathrm{m}\end{array}\right)$

and $\mathrm{UTU}^{-1}=\mathrm{T}$.

The potential energy matrix is

$$
\mathrm{V}=\left(\begin{array}{rrrrrr}
2 & -1 & 0 & 0 & 0 & -1 \\
-1 & 2 & -1 & 0 & 0 & 0 \\
0 & -1 & 2 & -1 & 0 & 0 \\
0 & 0 & -1 & 2 & -1 & 0 \\
0 & 0 & 0 & -1 & 2 & -1 \\
-1 & 0 & 0 & 0 & -1 & 2
\end{array}\right) \quad \text { and }
$$


$\mathrm{UVU}^{-1}=\left(\begin{array}{cccccc}2 & -2 & 0 & 0 & 0 & 0 \\ -2 & 2 & 0 & 0 & 0 & 0 \\ 0 & 0 & 2 & \left(-\frac{1}{2}+\frac{i \sqrt{3}}{2}\right) & 0 & 0 \\ 0 & 0 & \left(-\frac{1}{2}-\frac{i \sqrt{3}}{2}\right) & 2 & 0 & 0 \\ 0 & 0 & 0 & 0 & 2 & \left(-\frac{1}{2}-\frac{i \sqrt{3}}{2}\right) \\ 0 & 0 & 0 & 0 & \left(-\frac{1}{2}+\frac{i \sqrt{3}}{2}\right) & 2\end{array}\right)$

Thus, if $N \equiv \operatorname{col}\left(n_{1} n_{2} n_{3} n_{4} n_{5} n_{6}\right)$, and $N^{*} \equiv \operatorname{col}\left(n_{1}^{*} n_{2}^{*} n_{3}^{*} n_{4}^{*} n_{5}^{*} n_{6}^{*}\right)$, we have

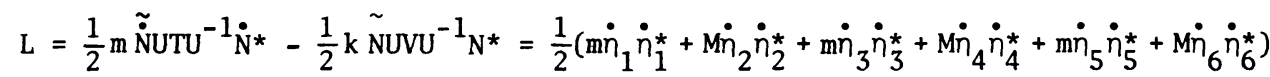$$
-\frac{1}{2} k\left(2 n_{1} n_{1}^{*}-2 n_{1} n_{2}^{*}-2 n_{2} n_{1}^{*}+2 n_{2} n_{2}^{*}+2 n_{3} n_{3}^{*}+\left(-\frac{1}{2}+\frac{i \sqrt{3}}{2}\right) n_{3} n_{4}^{*}+\left(-\frac{1}{2}-\frac{i \sqrt{3}}{2}\right) n_{4} n_{3}^{*}\right.
$$$$
\left.+2 n_{4} n_{4}^{*}+2 n_{5} n_{5}^{*}+\left(-\frac{1}{2}-\frac{i \sqrt{3}}{2}\right) n_{5} n_{6}^{*}+\left(-\frac{1}{2}+\frac{i \sqrt{3}}{2}\right) n_{6} n_{5}^{*}+2 n_{6} n_{6}^{*}\right) \text {. }
$$

The equations of motion are given by

$$
\frac{d}{d t}\left(\frac{\partial L}{\partial \eta_{j}^{*}}\right)-\frac{\partial L}{\partial \eta_{j}^{*}}=0 \quad, j \varepsilon\{1,2,3,4,5,6\} .
$$

Thus we have

$\ddot{n}_{1}=-\frac{2 k}{m} n_{1}+\frac{2 k}{m} n_{2}$ and $\ddot{n}_{2}=\frac{2 k}{M} n_{1}-\frac{2 k}{M} n_{2}$, implying natural frequencies $f_{1}=0$ and $f_{2}=\frac{1}{2 \pi} \sqrt{2 k\left(\frac{1}{m}+\frac{1}{M}\right)} ; \quad \ddot{n}_{3}=-\frac{2 k}{m} n_{3}+\frac{k}{2 m}(1+i \sqrt{3}) \eta_{4}$ and $\ddot{n}_{4}=\frac{k}{2 M}(1-i \sqrt{3}) n_{3}-\frac{2 k}{M} \eta_{4}$, implying natural frequencies $f_{3}=\frac{1}{2 \pi} \sqrt{k} \sqrt{\left(\frac{1}{m}+\frac{1}{M}\right)-\left(\frac{1}{m^{2}}-\frac{1}{M n}+\frac{1}{M^{2}}\right)^{1 / 2}} \quad$ and $f_{4}=\frac{1}{2 \pi} \sqrt{k} \sqrt{\left(\frac{1}{m}+\frac{1}{M}\right)+\left(\frac{1}{m^{2}}-\frac{1}{M m}+\frac{1}{M^{2}}\right)^{1 / 2}} ; \ddot{n}_{5}=-\frac{2 k}{m} n_{5}+\frac{k}{2 m}(1-i \sqrt{3}) \eta_{3}$ and $\ddot{n}_{6}=\frac{k}{2 M}(1+i \sqrt{3}) n_{5}-\frac{2 k}{M} n_{6}$, implying that $f_{5}=f_{3}$ and $f_{6}=f_{4}$. 
EXAMPLE 2. Three identical rods of length $l$ are pivoted about a fixed common axis at the centroid of an equilateral triangle so that the rods lie along the angle bisectors as shown. The ends of the rods which fall on the vertices of the triangle are connected with identical springs of force constant $k$. The moment of inertia with respect to the fixed axis is I for each rod. We want to compute frequencies for very small amplitude vibrations in the plane of the triangle.

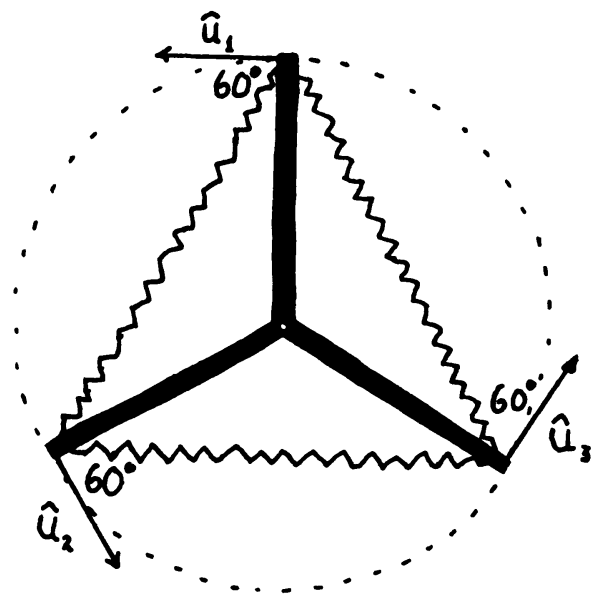

SOLUTION. The potential energy is given by

P.E. $=\frac{1}{2} k\left[\left(x_{1}-x_{2}\right)^{2}+\left(x_{2}-x_{3}\right)^{2}+\left(x_{3}-x_{1}\right)^{2}\right] \cos ^{2} 60^{\circ}=\frac{1}{2} k \tilde{x} V x$ where $\mathrm{X} \equiv \operatorname{col}\left(\mathrm{x}_{1} \mathrm{x}_{2} \mathrm{x}_{3}\right), \mathrm{x}_{\mathrm{j}}$ is the displacement of the vertex along $\hat{u}_{j}$, and 


$$
\mathrm{V}=\left|\begin{array}{rrr}
\frac{1}{2} & -\frac{1}{4} & -\frac{1}{4} \\
-\frac{1}{4} & \frac{1}{2} & -\frac{1}{4} \\
-\frac{1}{4} & -\frac{1}{4} & \frac{1}{2}
\end{array}\right|
$$

The kinetic energy is K.E. $=\frac{1}{2} I\left(\frac{\dot{x}_{1}^{2}+\dot{x}_{2}^{2}+\dot{x}_{3}^{2}}{\ell^{2}}\right)=\frac{1}{2}\left(\frac{I}{\ell^{2}}\right) \tilde{\dot{X}} T \dot{x}$ where

$$
T=\left|\begin{array}{lll}
1 & 0 & 0 \\
0 & 1 & 0 \\
0 & 0 & 1
\end{array}\right|
$$

The symmetry group is again taken to be $\mathrm{C}_{3}$ and the projection operators are as in the preceding example. Their action upon the basis $\left\{\hat{\mathrm{u}}_{1}, \hat{\mathrm{u}}_{2}, \hat{\mathrm{u}}_{3}\right\} \quad$ produces the symmetry coordinates

$$
\begin{aligned}
& \hat{\mathrm{e}}_{1}=\frac{1}{\sqrt{3}}\left(\hat{\mathrm{u}}_{1}+\hat{\mathrm{u}}_{2}+\hat{\mathrm{u}}_{3}\right), \hat{\mathrm{e}}_{2}=\frac{1}{\sqrt{3}}\left(\hat{\mathrm{u}}_{1}+\left(-\frac{1}{2}-\frac{i \sqrt{3}}{2}\right) \hat{\mathrm{u}}_{2}+\left(-\frac{1}{2}+\frac{i \sqrt{3}}{2}\right) \hat{\mathrm{u}}_{3}\right), \\
& \text { and } \hat{\mathrm{e}}_{3}=\frac{1}{\sqrt{3}}\left(\hat{\mathrm{u}}_{1}+\left(-\frac{1}{2}+\frac{i \sqrt{3}}{2}\right) \hat{\mathrm{u}}_{2}+\left(-\frac{1}{2}-\frac{i \sqrt{3}}{2}\right) \hat{\mathrm{u}}_{3}\right) \text {. Thus }
\end{aligned}
$$

$$
U=\frac{1}{\sqrt{3}}\left|\begin{array}{ccc}
1 & 1 & 1 \\
1 & \left(-\frac{1}{2}-\frac{i \sqrt{3}}{2}\right) & \left(-\frac{1}{2}+\frac{i \sqrt{3}}{2}\right) \\
1 & \left(-\frac{1}{2}+\frac{i \sqrt{3}}{2}\right) & \left(-\frac{1}{2}-\frac{i \sqrt{3}}{2}\right)
\end{array}\right|
$$


Next we obtain

$\mathrm{UTU}^{-1}=\mathrm{T}$ and $\mathrm{UVU}^{-1}=\left(\begin{array}{ccc}0 & 0 & 0 \\ 0 & \frac{3}{4} & 0 \\ 0 & 0 & \frac{3}{4}\end{array}\right)$

Then the Lagrangian is given by

$$
L=\frac{1}{2}\left(\frac{1}{\ell^{2}}\right)\left(\dot{n}_{1} \dot{n}_{1}^{*}+\dot{n}_{2} \dot{n}_{2}^{*}+\dot{n}_{3} \dot{n}_{3}^{*}\right)-\frac{1}{2} k\left(\frac{3}{4} n_{2} n_{2}^{*}+\frac{3}{4} n_{3} n_{3}^{*}\right)
$$

The equations of motion are

$\ddot{n}_{1}=0, \ddot{n}_{2}=-\frac{3 l^{2} k}{4 I} n_{2}$, and $\ddot{n}_{3}=-\frac{3 l^{2} k}{4 I} n_{3}$ implying the frequencies $f_{1}=0, f_{2}=f_{3}=\frac{\ell}{4 \pi} \sqrt{\frac{3 k}{I}}$.

Lastly, we present a problem similar to that in the first example.

EXAMPLE 3. Eight point masses of which four have the value $m$ and four have the value $M$ are symmetrically arranged about a fixed circle. The masses are interconnected with identical springs of force constant $k$ Motion is again confined to the circle and we wish to find the natural frequencies.

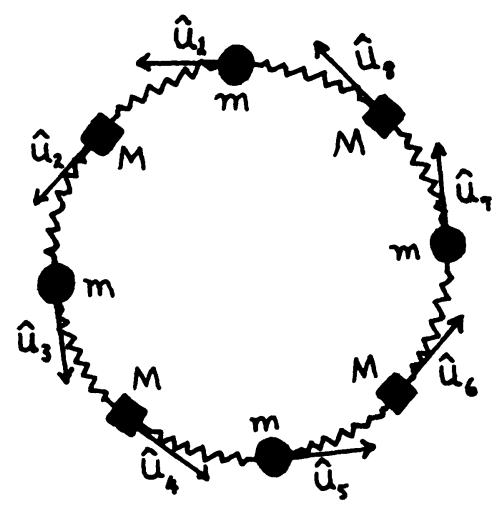


SOLUTION. If we take the symmetry group to be $C_{4}=\left\{E, R\left(90^{\circ}\right), R\left(180^{\circ}\right), R\left(270^{\circ}\right)\right\}$, there are four irreducible representations:

$$
\begin{aligned}
\Gamma^{(1)}\left(\mathrm{C}_{4}\right): \Gamma^{(1)}(\mathrm{g})=1 \forall \mathrm{g \varepsilon C}_{4} ; \\
\Gamma^{(2)}\left(\mathrm{C}_{4}\right): \Gamma^{(2)}(\mathrm{E})=\Gamma^{(2)}\left(\mathrm{R}\left(180^{\circ}\right)\right)=1, \\
\Gamma^{(2)}\left(\mathrm{R}\left(90^{\circ}\right)\right)=\Gamma^{(2)}\left(\mathrm{R}\left(270^{\circ} \mathrm{j}\right)=-1 ;\right. \\
\Gamma^{(3)}\left(\mathrm{C}_{4}\right): \Gamma^{(3)}(\mathrm{E})=1, \Gamma^{(3)}\left(\mathrm{R}\left(90^{\circ}\right)\right)=\mathrm{i}, \\
\Gamma^{(3)}\left(\mathrm{R}\left(180^{\circ}\right)\right)=-1, \Gamma^{(3)}\left(\mathrm{R}\left(270^{\circ}\right)\right)=-i ; \\
\Gamma^{(4)}\left(\mathrm{C}_{4}\right): \Gamma^{(4)}(\mathrm{E})=1, \Gamma^{(4)}\left(\mathrm{R}\left(90^{\circ}\right)\right)=-\mathrm{i}, \\
\Gamma^{(4)}\left(\mathrm{R}\left(180^{\circ}\right)\right)=-1, \Gamma^{(4)}\left(\mathrm{R}\left(270^{\circ}\right)\right)=i
\end{aligned}
$$

There are four projection operators which determine the transformation matrix $U$. They are

$$
\begin{aligned}
& P_{11}^{(1)}=\sum_{g \varepsilon C_{4}} \Gamma^{(1)}(g) \phi(g), \\
& P_{11}^{(2)}=\sum_{g \varepsilon C_{4}} \Gamma^{(2)}(g) \phi(g), \\
& P_{11}^{(3)}=\sum_{g \in C_{4}} \Gamma^{(3)}(g) \phi(g), \text { and } \\
& P_{11}^{(4)}=\sum_{g \varepsilon C_{4}} \Gamma^{(4)}(g) \phi(g),
\end{aligned}
$$

After the projection operators are applied to each unit tangent vector, the resulting, distinct, nonzero vectors are normalized to give the rows in the matrix $U$. Thus 


$$
U=\frac{1}{2} \quad\left(\begin{array}{cccccccc}
1 & 0 & 1 & 0 & 1 & 0 & 1 & 0 \\
0 & 1 & 0 & 1 & 0 & 1 & 0 & 1 \\
1 & 0 & -1 & 0 & 1 & 0 & -1 & 0 \\
0 & 1 & 0 & -1 & 0 & 1 & 0 & -1 \\
1 & 0 & -i & 0 & -1 & 0 & i & 0 \\
0 & 1 & 0 & -i & 0 & -1 & 0 & i \\
1 & 0 & i & 0 & -1 & 0 & -i & 0 \\
0 & 1 & 0 & i & 0 & -1 & 0 & -i
\end{array}\right)
$$

The Lagrangian is $L=\frac{1}{2} m \tilde{\dot{X}} T \dot{X}-\frac{1}{2} k \tilde{X} V X$ where $x \equiv c o l\left(x_{1} x_{2} x_{3} x_{4} x_{5} x_{6} x_{7} x_{8}\right)$, $\mathrm{T}=\left(\begin{array}{cccccccc}1 & 0 & 0 & 0 & 0 & 0 & 0 & 0 \\ 0 & \mathrm{M} / \mathrm{m} & 0 & 0 & 0 & 0 & 0 & 0 \\ 0 & 0 & 1 & 0 & 0 & 0 & 0 & 0 \\ 0 & 0 & 0 & \mathrm{M} / \mathrm{m} & 0 & 0 & 0 & 0 \\ 0 & 0 & 0 & 0 & 1 & 0 & 0 & 0 \\ 0 & 0 & 0 & 0 & 0 & \mathrm{M} / \mathrm{m} & 0 & 0 \\ 0 & 0 & 0 & 0 & 0 & 0 & 1 & 0 \\ 0 & 0 & 0 & 0 & 0 & 0 & 0 & \mathrm{M} / \mathrm{m}\end{array}\right)$, and $\mathrm{V}=\left(\begin{array}{rrrrrrrr}2 & -1 & 0 & 0 & 0 & 0 & 0 & -1 \\ -1 & 2 & -1 & 0 & 0 & 0 & 0 & 0 \\ 0 & -1 & 2 & -1 & 0 & 0 & 0 & 0 \\ 0 & 0 & -1 & 2 & -1 & 0 & 0 & 0 \\ 0 & 0 & 0 & -1 & 2 & -1 & 0 & 0 \\ 0 & 0 & 0 & 0 & -1 & 2 & -1 & 0 \\ 0 & 0 & 0 & 0 & 0 & -1 & 2 & -1 \\ -1 & 0 & 0 & 0 & 0 & 0 & -1 & 2\end{array}\right)$

Then $\mathrm{UTU}^{-1}=\mathrm{T}$ and 
$\mathrm{UVU}^{-1}=\left(\begin{array}{cccccccc}2 & -2 & 0 & 0 & 0 & 0 & 0 & 0 \\ -2 & 2 & 0 & 0 & 0 & 0 & 0 & 0 \\ 0 & 0 & 2 & 0 & 0 & 0 & 0 & 0 \\ 0 & 0 & 0 & 2 & 0 & 0 & 0 & 0 \\ 0 & 0 & 0 & 0 & 2 & (-1+i) & 0 & 0 \\ 0 & 0 & 0 & 0 & (-1-i) & 2 & 0 & 0 \\ 0 & 0 & 0 & 0 & 0 & 0 & 2 & (-1-i) \\ 0 & 0 & 0 & 0 & 0 & 0 & (-1+i) & 2\end{array}\right)$.

The transformed Lagrangian is

$$
\begin{aligned}
L & =\frac{1}{2}\left(m \dot{n}_{1} \dot{n}_{1}^{*}+M_{2} \dot{n}_{2}^{*}+\dot{m}_{3} \dot{n}_{3}^{*}+M_{n_{4}} \dot{n}_{4}^{*}+\dot{m}_{5} \dot{n}_{5}^{*}+M_{6} \dot{n}_{6}^{*}+\dot{m}_{7} \dot{n}_{7}^{*}+M_{n_{8}} \dot{n}_{8}^{*}\right) \\
& -\frac{1}{2} k\left(2 n_{1} n_{1}^{*}-2 n_{2} n_{1}^{*}-2 n_{1} n_{2}^{*}+2 n_{2} n_{2}^{*}+2 n_{3} n_{3}^{*}+2 n_{4} n_{4}^{*}+2 n_{5} n_{5}^{*}\right. \\
& +(-1-i) n_{6} n_{5}^{*}+(-1+i) n_{5} n_{6}^{*}+2 n_{6} n_{6}^{*}+2 n_{7} n_{7}^{*}+(-1+i) n_{8} n_{7}^{*} \\
& \left.+(-1-i) n_{7} n_{8}^{*}+2 n_{8} n_{8}^{*}\right) .
\end{aligned}
$$

The equations of motion and natural frequencies are found in the standard manner with the results that

$$
\begin{aligned}
& \mathrm{f}_{1}=0, \mathrm{f}_{2}=\frac{1}{2 \pi} \sqrt{2 \mathrm{k}\left(\frac{1}{\mathrm{~m}}+\frac{1}{\mathrm{M}}\right)}, \mathrm{f}_{3}=\frac{1}{2 \pi} \sqrt{\frac{2 \mathrm{k}}{\mathrm{m}}}, \mathrm{f}_{4}=\frac{1}{2 \pi} \sqrt{\frac{2 \mathrm{k}}{\mathrm{M}}}, \\
& \mathrm{f}_{5}=\mathrm{f}_{7}=\frac{1}{2 \pi} \sqrt{\mathrm{k}} \sqrt{\left(\frac{1}{\mathrm{~m}}+\frac{1}{\mathrm{M}}\right)+\left(\frac{1}{\mathrm{~m}^{2}}+\frac{1}{\mathrm{M}^{2}}\right)^{1 / 2}}, \text { and } \\
& \mathrm{f}_{6}=\mathrm{f}_{8}=\frac{1}{2 \pi} \sqrt{\mathrm{k}} \sqrt{\left(\frac{1}{\mathrm{~m}}+\frac{1}{\mathrm{M}}\right)-\left(\frac{1}{\mathrm{~m}^{2}}+\frac{1}{\mathrm{M}^{2}}\right)^{1 / 2}} .
\end{aligned}
$$

\section{REFERENCES}

1. Boyd, J.N. and P.N. Raychowdhury, "An Application of Projection Operators to a One Dimensional Crystal," Bulletin of the Institute of Mathematics, Academia Sinica 7 (1979), 133-144. 
2. Duffey, G.H., Theoretical Physics, Houghton Mifflin Company, Boston, 1973 .

3. Gamba, A., "Representations and Classes in Groups of Finite Order," Journal of Mathematical Physics 9 (1968), 186-192.

4. Hamermesh, M., Group Theory and Its Application to Physical Problems, Addison-Wesley Publishing-Company, Reading, Mass., 1962.

5. Joshi, A.W., Elements of Group Theory for Physicists, Halsted Press, New York, 1973.

6. Nussbaum, A., "Group Theory and Normal Modes," American Journal of Physics 36 (1968), 529-539.

7. Wilde, R.E., "A General Theory of Symmetry Coordinates," American Journal of Physics 32 (1964), 45-52. 


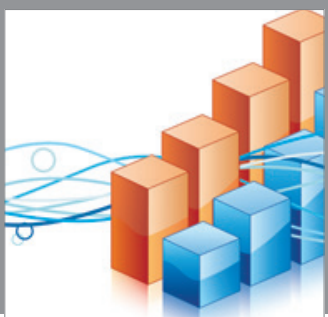

Advances in

Operations Research

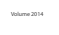

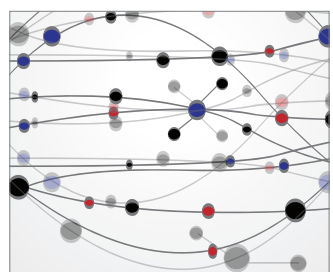

\section{The Scientific} World Journal
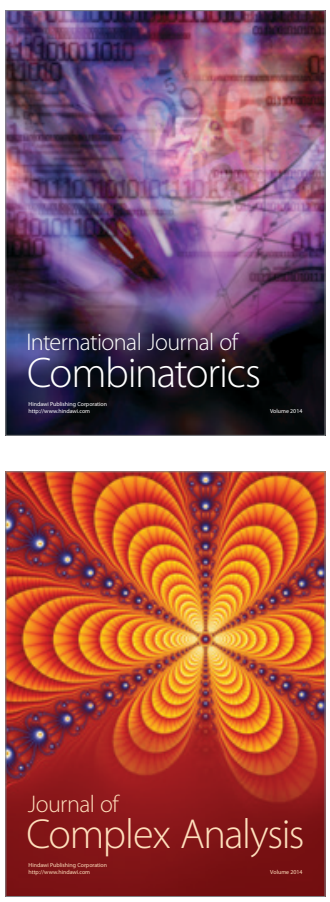

International Journal of

Mathematics and

Mathematical

Sciences
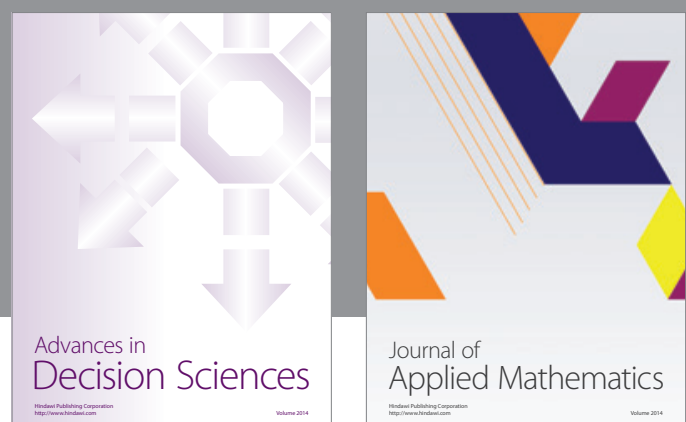

Journal of

Applied Mathematics
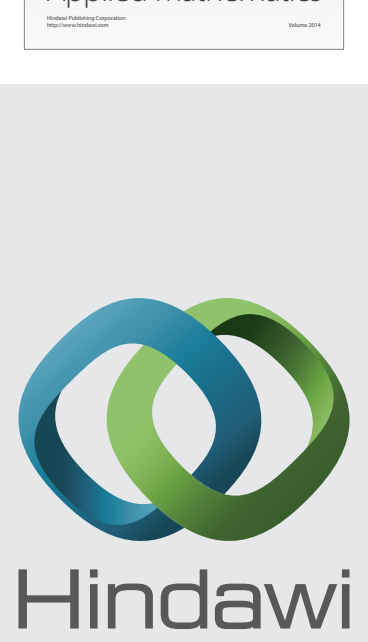

Submit your manuscripts at http://www.hindawi.com
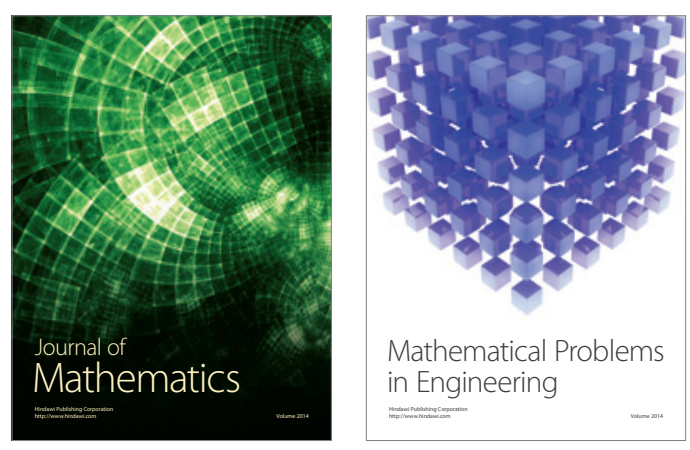

Mathematical Problems in Engineering
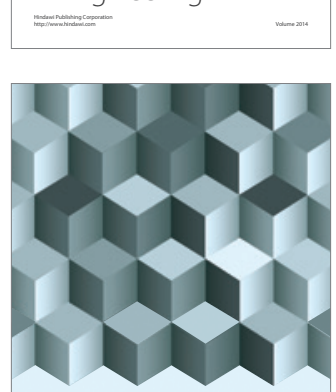

Journal of

Function Spaces
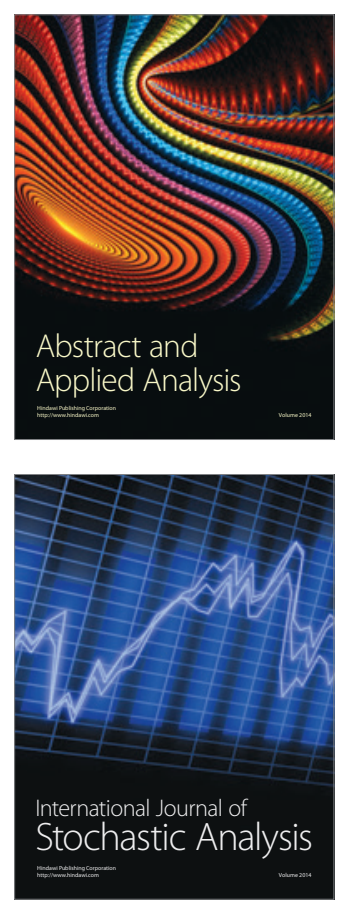

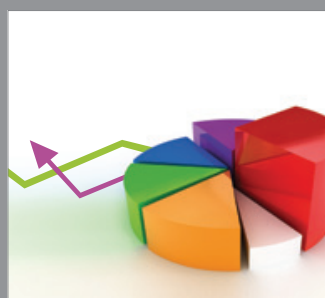

ournal of

Probability and Statistics

Promensencen
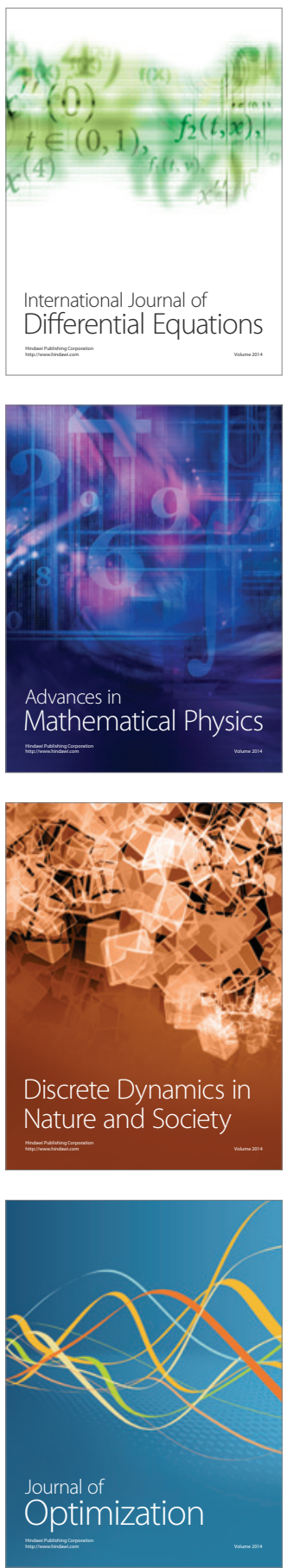